\title{
High-throughput and data-mining approach to predict new rare-earth free permanent magnets
}

\author{
Alena Vishina $\odot,{ }^{1, *}$ Olga Yu. Vekilova, ${ }^{1}$ Torbjörn Björkman $\odot,{ }^{2}$ Anders Bergman, ${ }^{1}$ Heike C. Herper $\odot,{ }^{1}$ and Olle Eriksson ${ }^{1,3}$ \\ ${ }^{1}$ Department of Physics and Astronomy, Uppsala University, Box 516, SE-75120, Uppsala, Sweden \\ ${ }^{2}$ Department of Natural Sciences, Åbo Akademi, FI-20500 Turku, Finland \\ ${ }^{3}$ School of Science and Technology, Örebro University, SE-701 82 Örebro, Sweden
}

(Received 2 October 2019; revised manuscript received 15 January 2020; accepted 4 February 2020; published 3 March 2020)

\begin{abstract}
We present an application of a high-throughput search of new rare-earth free permanent magnets focusing on $3 d-5 d$ transition metal compounds. The search involved a part of the Inorganic Crystal Structure Database, together with tailored search criteria and electronic structure calculations of magnetic properties. Our results suggest that it is possible to find candidates for rare-earth free permanent magnets using a data-mining/datafiltering approach. The most promising candidates identified here are $\mathrm{Pt}_{2} \mathrm{FeNi}, \mathrm{Pt}_{2} \mathrm{FeCu}$, and $\mathrm{W}_{2} \mathrm{FeB}$. We suggest these materials to be a good platform for further investigations in the search of novel rare-earth free permanent magnets.
\end{abstract}

DOI: 10.1103/PhysRevB.101.094407

\section{INTRODUCTION}

High performance permanent magnets are needed for a large number of applications, such as electric vehicle motors and generators, wind mills, loud speakers, and relays. The materials' properties needed for these applications, many of which represent a "green" technology of energy conversion, are an essentially high saturation magnetization, a large Curie temperature, and a large magnetic anisotropy. Most permanent magnets that are used today are either ferrites [1] or rare-earth (RE) containing compounds, such as $\mathrm{Nd}_{2} \mathrm{Fe}_{14} \mathrm{~B}$ [2]. In fact, most high performance magnets contain RE materials ( $\mathrm{Pr}$, $\mathrm{Nd}, \mathrm{Sm}, \mathrm{Tb}$, or Dy) [3], which makes them expensive while some of the RE elements (like Dy) are rapidly decreasing in availability and therefore bear an economic risk. With the increase in the number of electric vehicles and the usage of wind power generators, there is a growing need to reduce or eliminate the usage of RE magnets by finding rare-earth free alternatives, which at the same time are expected to at least show the same price performance. Due to the high interest in the field, various materials [4-6], nanostructures [7-10], thin films $[11,12]$, and new compounds have been proposed. In addition, new techniques such as machine learning [13] have been suggested in the quest for finding RE-free permanent magnets. Most of the papers devoted to this effort describe the experimental investigations of a single compound, or a smaller group of materials with similar structure [4,5,14-20].

*Present address: Department of Physics and Astronomy, Uppsala University, Box 516, SE-75120, Uppsala, Sweden; alena.vishina@physics.uu.se

Published by the American Physical Society under the terms of the Creative Commons Attribution 4.0 International license. Further distribution of this work must maintain attribution to the author(s) and the published article's title, journal citation, and DOI. Funded by Bibsam.
Experimental studies of the magnetic properties of a single material require first synthesizing it and performing a number of measurements to characterize the required quantities; e.g., crystal structure, saturation- and remanent magnetic moments, coercive field, and ordering temperature. This is a cumbersome and time-consuming effort that makes a systematic study of a large number of materials, not only expensive, but also extremely lengthy. Even though there exist experimental high-throughput studies, a detailed analysis of materials' properties as described above can be extremely time consuming. Theoretical calculations, especially based on density functional theory (DFT), have developed at a rapid speed and are now found to reproduce essentially all magnetic properties that are needed for a suitable permanent magnet [21]. In fact, there are even a few cases when theory has made predictions of materials with suitable characteristics as RE-free permanent magnets. For instance, Fe-Co alloys that were tuned to a specific concentration and tetragonal strain were predicted to have a large magnetic moment and magnetic anisotropy [14], and subsequent experimental work [15] confirmed this suggestion. It should be noted here that the most challenging aspect of these studies is the calculation of the magnetic anisotropy energy (MAE) due to its extremely small value. However, since the pioneering works of Brooks [22] several attempts [23-27] have shown that, with few exceptions, theory reproduces the easy axis orientation and the right order of magnitude of the MAE. Hence, theory is a good alternative that allows to identify new phases and compounds that are suitable candidates for RE-free permanent magnets. We therefore combined density functional theory, a high-throughput search from the Inorganic Crystal Structure Database (ICSD) [28], and filtering through a large amount of data to identify the new RE-free materials that can be proposed as new permanent magnets.

A similar approach has recently been applied for crystal structure predictions $[29,30]$, in the search of materials with specific properties [31-33], such as new 
two-dimensional materials [34,35], new perovskites [36], possible high-temperature superconductors [37,38], new scintillator materials [39], topological insulators [40], spin-gapless semiconductors [41], magnetic materials among the Heusler alloys [42], and materials design in general $[43,44]$. From our investigations we identified three compounds that fulfill the criteria for being suitable as permanent magnets, without containing RE elements.

\section{COMPUTATIONAL METHODS}

An initial screening was performed using a database of about 10000 calculations derived from the compounds in the ICSD containing the elements $\mathrm{Cr}, \mathrm{Mn}, \mathrm{Fe}, \mathrm{Co}$, and $\mathrm{Ni}$, and which have no more than 16 atoms in their primitive unit cell. These calculations were performed using the full-potential linear muffin-tin orbital method (FP-LMTO), including spinorbit interaction as implemented in the RSPT code $[45,46]$, with the AM05 functional [47] for exchange and correlation. The calculation time depended strongly on the system and varied between several and several hundreds CPU hours. For the high-throughput screening the initial setup for all the materials was ferromagnetic.

To determine the magnetic ground state of materials, i.e., ferromagnetic (FM) or antiferromagnetic (AFM), Vienna $A b$ Initio Simulation Package (VASP) [48-51] was used within the projector augmented wave (PAW) method [52], along with the generalized gradient approximation (GGA) in Perdew, Burke, and Ernzerhof (PBE) form [53].

For the phase stability check of the materials the differences in enthalpies were calculated between the systems and proper stable binary compounds chosen from the corresponding phase diagrams. For instance, the stability of $\mathrm{Pt}_{2} \mathrm{FeNi}$ is checked by calculating the formation enthalpy in the form

$$
\Delta H=H_{\mathrm{FeNiPt}_{2}}-H_{\mathrm{FePt}}-H_{\mathrm{NiPt}},
$$

where $H_{\mathrm{FePt}}$ and $H_{\mathrm{NiPt}}$ are the enthalpies of $\mathrm{FePt}$ and NiPt, respectively.

The MAE calculations were done with the the RSPT code, as $\Delta E=E^{p l}-E^{c}$, where $E^{c}$ and $E^{p l}$ are the total energies with the magnetization directed along and perpendicular to the $c$-axis. The results were obtained with the tetrahedron method with Blöchl correction for the Brillouin zone integration [54]; the converged $k$-point Monkhorst-Pack meshes [55] between $28 \times 28 \times 28$ and $34 \times 34 \times 34$ were used for the calculations depending on the material. Materials with the positive $\Delta E$ have uniaxial magnetic anisotropy required for a permanent magnet. Calculating the MAE depending on the material required CPU time between several hundreds and several thousands hours.

The magnetic hardness parameter was calculated as $\kappa=$ $\sqrt{\Delta E / \mu_{0} M_{S}^{2}}$ [56], where $M_{S}$ is saturation magnetization and $\mu_{0}$ is the vacuum permeability. This parameter is often used to identify promising permanent magnets. Its relevance comes about since, in practice, a large coercive field is needed for a good permanent magnet. A large MAE is, however, a prerequisite for permanent magnets.

The Curie temperature was estimated using Monte Carlo simulations implemented within Uppsala atomistic spin dynamics (UPPASD) software [21] starting from the magnetic parameters computed with the RSPT code. The ASD simulations were performed on a $35 \times 35 \times 35$ supercell, with periodic boundary conditions, using the exchange parameters calculated with RSPT code within the first nine coordination shells.

The SUMO package [57] was used for the density of states (DOS) plots.

\section{HIGH-THROUGHPUT AND SCREENING}

There are several criteria which are necessary for a material to be a strong permanent magnet. Among these are ferromagnetic ordering, high saturation magnetization $\left(M_{S}>1 \mathrm{~T}\right)$, high Curie temperature $\left(T_{\mathrm{C}}>400^{\circ} \mathrm{C}\right)$, and, more importantly, the large uniaxial magnetic anisotropy energy (MAE) $\left(>1 \mathrm{MJ} / \mathrm{m}^{3}\right)$. The most widely used rare-earth permanent magnets $\mathrm{Nd}_{2} \mathrm{Fe}_{14} \mathrm{~B}$ and $\mathrm{Sm}_{2} \mathrm{Co}_{17}$ have $\mathrm{MAE}=5 \mathrm{MJ} / \mathrm{m}^{3}$ and $3.3 \mathrm{MJ} / \mathrm{m}^{3}$, saturation magnetization $M_{S}=1.6 \mathrm{~T}$ and $M_{S}=$ $1.3 \mathrm{~T}$, and Curie temperature of 585 and $1100 \mathrm{~K}$, respectively [58-60]. Even though a large magnetization is desirable for permanent magnets, the ratio between magnetization and MAE is even more important since it determines the hardness $\kappa$ of a magnet and $\kappa \geqslant 1$ is needed for a hard magnet in order to resist the self-demagnetization [2].

Evaluating the saturation magnetization from firstprinciples theory is rather straight-forward, and can be done for a large number of materials in a relatively efficient manner. The calculation of MAE, on the other hand, is rather complex and involves an extremely small energy differences of the order of $\mu \mathrm{eV}[27,61]$. The magnetic anisotropy is the result of relativistic effects, primarily the spin-orbit coupling, combined with the exchange splitting of the energy bands, and hence requires first-principles calculations using a relativistic formulation of the Kohn-Sham equations.

The search presented here was performed in two parts (see Fig. 1): first, by performing a high-throughput screening of a very large number of possible compounds a limited list of materials was identified, and then more detailed calculations were used for the next part. The workflow of this approach was such that initially a high-throughput screening was made, using data sets provided by the ICSD [28], for which we applied a set of initial screening parameters. In the present study we report on results of a search among compounds that have at least one $3 d$ and one $5 d$ element. Such materials have the potential to have a significant MAE since the $3 d$ element would provide significant exchange interaction and magnetic ordering temperature, while the $5 d$ element would guarantee the large spin-orbit coupling that is needed for the MAE. This initial choice left us with a list of more than a thousand systems.

After the first principles calculations (see the Computational Methods section, Sec. II) of the materials identified in the initial step were made, a further screening step was introduced, where we considered only materials with calculated magnetic moment higher than $0.5 \mu_{B} /$ f.u. Several hundreds systems were left that satisfy that condition. To decrease the number of systems before the more computationally costly steps, several screening conditions were introduced that did not rely on any theoretical calculations, only information from the ICSD [28] database was used. Since it is known that 


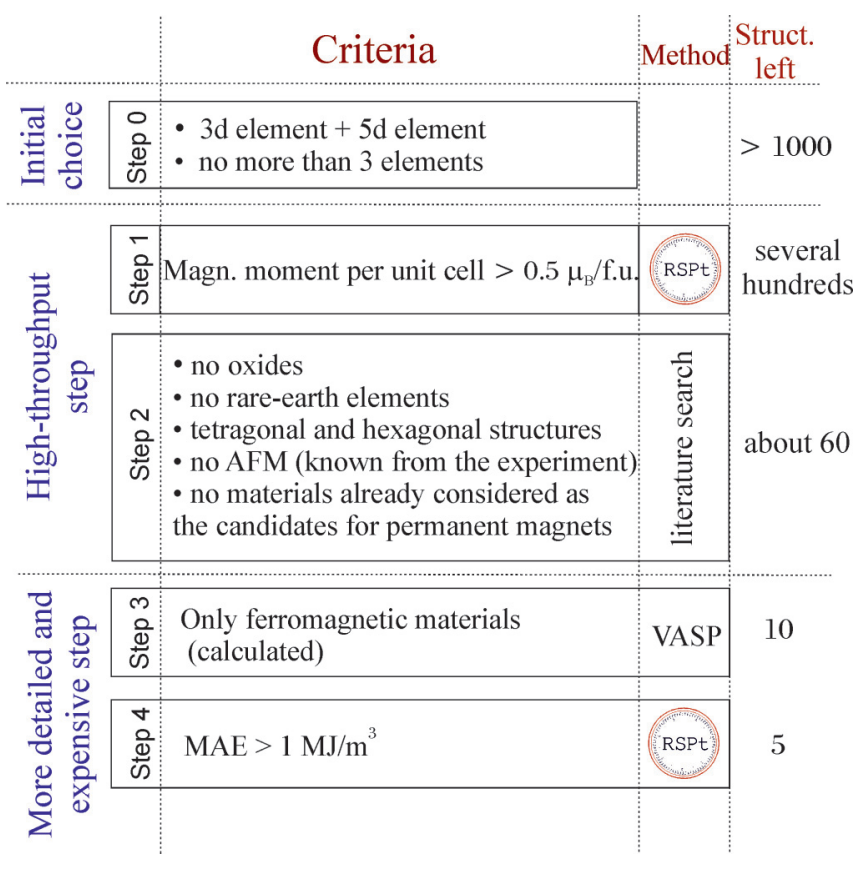

FIG. 1. Steps of high-throughput search and screening with the number of structures left after each step.

spin-orbit effects influence the MAE most effectively for noncubic materials, we screened to identify tetragonal and hexagonal materials, and considered them only for the further steps.

Among these compounds several oxide materials were identified. Since transition metal oxides often have a correlated electronic structure in such a way that single determinant theories such as density functional theory fail, we excluded these compounds. It should be emphasized that these oxides were excluded not because they were considered uninteresting for applications as permanent magnets, but because the theory employed here is known to have questionable accuracy when it comes to describing magnetic properties, something which, in particular, applies to the MAE. All materials discarded at this "theoretical" step were set aside for the future consideration.

To further reduce the number of compounds that were subjected to the careful, high precision calculations of the saturation moment and MAE, we excluded the materials consisting of more than three elements. All these screening steps left us with less than 60 possible candidates. Some, but not all, of these compounds were previously investigated with regard to their magnetic properties, at least the magnetic structure has been reported for some of them. All the materials that are known in the literature to be antiferromagnetic or paramagnetic (PM) at room temperature were then excluded from the list, which reduced the number of potential compounds to 25 . These systems were selected for high-precision calculations of the magnetic anisotropy and the ordering temperature, giving information about the four most important intrinsic magnetic properties of potential new permanent magnets-ferromagnetic ordering, large saturation moment, significant ordering temperature, and a relatively large magnetic anisotropy.
TABLE I. Well-known materials with appropriate magnetic characteristics for permanent magnet applications that came out of the screening steps proposed in this investigation. Listed are the ICSD database number, space group, MAE, magnetic moment $(\mu)$ per $3 \mathrm{~d}$ atom, and Curie temperature $\left(T_{\mathbf{C}}\right)$. In parentheses we note measured (exp) or previously calculated (th) data. References to previous work are also included in parentheses. $T_{\mathbf{C}}$ for $\mathrm{Fe}_{2} \mathrm{~W}$ was calculated in the present investigation.

\begin{tabular}{lcccccc}
\hline \hline \multicolumn{2}{c}{$\begin{array}{c}\text { ICSD } \\
\text { Material }\end{array}$ number } & Space & gAE (exp) & MAE (th) & $\mu$ & $T_{\mathrm{C}}$ \\
$\mathrm{MJ} / \mathrm{m}^{3}$ & $\mathrm{MJ} / \mathrm{m}^{3}$ & $\mu_{B} / 3 \mathrm{~d}$ & $\mathrm{~K}$ \\
\hline $\mathrm{Au}_{4} \mathrm{Mn}$ & 657182 & 87 & $0.3[62]$ & & $4.3[62]$ & $370[62]$ \\
$\mathrm{CoPt}$ & 197572 & 123 & $4.0[63]$ & $6.6[64]$ & $1.76[65]$ & $853[63]$ \\
& & & $3.0[65]$ & $6.9[66]$ & & \\
& & & $4.5[67]$ & & & \\
$\mathrm{FePt}$ & 633191 & 123 & $10.0[68]$ & $11.0[64]$ & $2.8[70]$ & $773[68]$ \\
$\mathrm{Fe}_{2} \mathrm{~W}$ & 634058 & 194 & & $0.87[69]$ & $0.43[69]$ & 320 \\
\hline \hline
\end{tabular}

\section{RESULTS}

\section{A. Findings from high-throughput calculations}

We applied the screening steps outlined above to materials that contain necessarily one $3 d$ and one $5 d$ element, while at the same time allowing the material to have no more than three elements in total. All the crystallographic data were taken from the ICSD [28]. The screening steps employed here allowed to single out a number of materials that have already been suggested and investigated as systems with high magnetic anisotropy in previous works [62-69]. In Table I we list the magnetic properties of these compounds. Note that several of the compounds in Table I have previously been recognized from theoretical and/or experimental work to have promising materials properties for applications as permanent magnets, e.g., FePt. Hence the fact that the screening method employed here identifies these systems renders credibility to its power as a predictive tool. We note here that of the compounds listed in Table $\mathrm{I}, \mathrm{Fe}_{2} \mathrm{~W}$ has previously been investigated only by theoretical means. The Curie temperature $T_{\mathbf{C}}$ was calculated for this material since it has not been reported before. A final comment on Table I is that when a comparison can be made, experiment and theory agree on the order of magnitude of the MAE as well as the easy axis. Very few examples exist in the literature that do not follow this trend.

Unfortunately, none of the materials listed in Table I is suitable for applications that need large mass/volume since they involve costly elements. The exception to this is $\mathrm{Fe}_{2} \mathrm{~W}$, but for this compound the saturation moment is too low to make it interesting for applications. However, alloying of this compound may improve the saturation moment. Such an investigation is outside the scope of the present work.

In addition to the known structures listed in Table I, a set of hitherto unexplored compounds was identified, which involved about 20 materials. For these compounds, more detailed and accordingly time-consuming considerations were made, where, in particular, focus was paid to a large magnetocrystalline anisotropy with uniaxial orientation, ferromagnetic ordering, high saturation magnetization, and large exchange interaction. The last guarantees a sufficiently high Curie temperature. Of these compounds, several failed to 


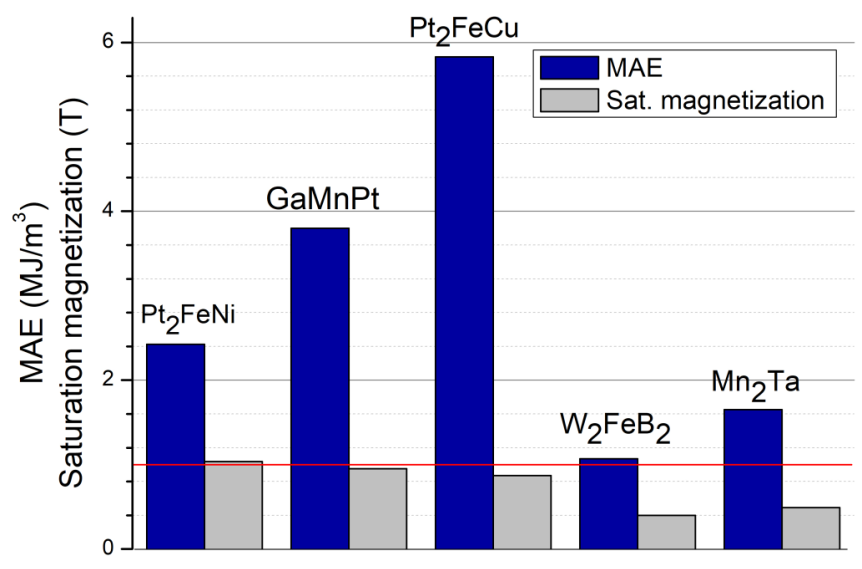

FIG. 2. MAE and saturation magnetization of several compounds that have not been investigated for magnetocrystalline anisotropy before (apart from $\mathrm{Pt}_{2} \mathrm{FeNi}$ that which was studied in the form of $\mathrm{L}_{0}$ alloy films [71]). The red line shows $\mathrm{MAE}=1 \mathrm{MJ} / \mathrm{m}^{3}$ and $M_{s}=1 \mathrm{~T}$ that are considered the necessary criteria of a strong permanent magnet.

match one or more of the above-mentioned key requirements for permanent magnets. The calculations showed that some of them have an antiferromagnetic or nonmagnetic (NM) ground state. Examples of such systems are $\mathrm{W}_{2} \mathrm{MnB}_{2}$, NiMnAs, GeMnTa, and $\mathrm{Co}_{2} \mathrm{Ta}$ (see the Appendices, Table IV, for the full list of materials we calculated to be AFM or NM). Materials that were found to have a planar easy axis $(\Delta E<0)$ or a MAE that is not sufficiently high were also excluded from the final list of proposed rare-earth free permanent magnets. Examples of systems that were put aside are $\mathrm{Al}_{8} \mathrm{Fe}_{4} \mathrm{Hf}$ and $\mathrm{Fe}_{7} \mathrm{~W}_{6}$. Although these materials are not identified here as having suitable magnetic properties for permanent magnet applications, we note that alloying of these systems, or investigations of the temperature dependence of the MAE, may render them suitable. However, such studies are outside the scope of the present investigation.

Apart from the previously known materials with good magnetic properties for the permanent magnet applications, listed above, five additional promising compounds were identified, see Fig. 2. To our knowledge the magnetic properties of these materials have not been investigated in detail before. These systems seem to possess magnetic properties that are required to be considered as a candidate phase for permanent magnets. The calculated magnetocrystalline anisotropy is uniaxial and well above the threshold of $1 \mathrm{MJ} / \mathrm{m}^{3}$. For three of them also the saturation magnetization is larger than $1 \mathrm{~T}$, only $\mathrm{Mn}_{2} \mathrm{Ta}$ and $\mathrm{W}_{2} \mathrm{FeB}_{2}$ fall behind with magnetization values around $0.5 \mathrm{~T}$, see Fig. 2. It may be noted here that $\mathrm{Pt}_{2} \mathrm{FeCu}$ has an extremely large MAE of about $6 \mathrm{MJ} / \mathrm{m}^{3}$. In fact, few materials can compete with such a large uniaxial anisotropy. Among the rare-earth free permanent magnets only materials such as $\mathrm{FePt}$ and CoPt provide MAE values of the same order of magnitude, see Table I. The MAE of GaMnPt is also very significant and is interesting from a practical point of view since it has even lower Pt concentration than the tetragonal $\mathrm{Pt}_{2} \mathrm{FeCu}$ system.

Taking a closer look at the systems in Fig. 2, by investigating the magnetic exchange parameters and magnetic
TABLE II. Calculated MAE, saturation magnetization, Curie temperature, and magnetic hardness parameter for materials that previously have not been investigated with respect to the magnetocrystalline anisotropy (apart from $\mathrm{Pt}_{2} \mathrm{FeNi}$, which was studied in the form of $\mathrm{L1}_{0}$ alloy films [71]).

\begin{tabular}{lcccccc}
\hline \hline Material & $\begin{array}{c}\text { ICSD } \\
\text { number }\end{array}$ & $\begin{array}{c}\text { Space } \\
\text { group }\end{array}$ & $\begin{array}{c}\text { MAE (th) } \\
\mathrm{MJ} / \mathrm{m}^{3}\end{array}$ & $\begin{array}{c}\text { Sat. magn. } \\
\mathrm{T}\end{array}$ & \multicolumn{1}{c}{$T_{\mathrm{C}}$} & \\
$\mathrm{K}$ & $\kappa$ \\
$\mathrm{Pt}_{2} \mathrm{FeNi}$ & 42564 & 123 & 2.42 & 1.04 & 230 & 1.68 \\
$\mathrm{Pt}_{2} \mathrm{FeCu}$ & 53259 & 123 & 5.83 & 0.87 & 30 & 3.11 \\
$\mathrm{~W}_{2} \mathrm{FeB}$ & 43016 & 127 & 1.07 & 0.40 & 165 & 2.90 \\
\hline \hline
\end{tabular}

coupling, revealed that GaMnPt and $\mathrm{Mn}_{2} \mathrm{Ta}$ have a more complex magnetic order and are, strictly speaking, not ferromagnets. Despite GaMnPt being found to be ferromagnetic at finite temperatures by several experimental groups [72-75] we found that in the $a b$ initio calculations it shows deviations from the collinear configuration. We believe that the observed ferromagnetic state of $\mathrm{GaMnPt}$ is possibly a result of being careful and tailored alloying, the investigation of lattice defects, or just as a finite temperature effect. However, to study this further is outside of the scope of this paper. Therefore, these two compounds are excluded from the suggested list of new materials, and their magnetic properties will be discussed elsewhere. For the remaining three tetragonal phases all calculated magnetic properties are summarized in Table II. The unit cells of these materials can be seen in Figs. 3 and 4. $\mathrm{Pt}_{2} \mathrm{FeCu}$ and $\mathrm{Pt}_{2} \mathrm{FeNi}$ have the same crystal structure, with the same site occupied either by $\mathrm{Cu}$ or by $\mathrm{Ni}$. Additional structural information can be found in Appendix B, Table V.

One of the materials listed in Table II, $\mathrm{Pt}_{2} \mathrm{FeNi}$, was previously studied in the form of $\mathrm{L}_{0}$ alloy films $\left.[71,76]\right)$. This system was found to have an experimental uniaxial magnetic anisotropy of $0.9 \mathrm{MJ} / \mathrm{m}^{3}$, which qualitatively agrees with the theoretical data presented here of bulk $\mathrm{Pt}_{2} \mathrm{FeNi}$ (see Table II).

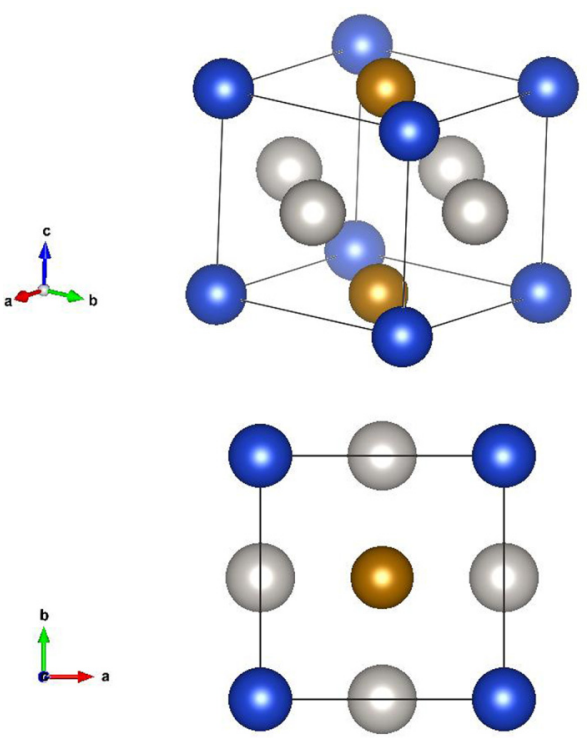

FIG. 3. Tetragonal unit cell of $\mathrm{Pt}_{2} \mathrm{FeM}(\mathrm{M}=\mathrm{Cu}, \mathrm{Ni})$. Iron atoms are shown with brown spheres, $\mathrm{M}$ atoms are blue, and $\mathrm{Pt}$ atoms are grey. 


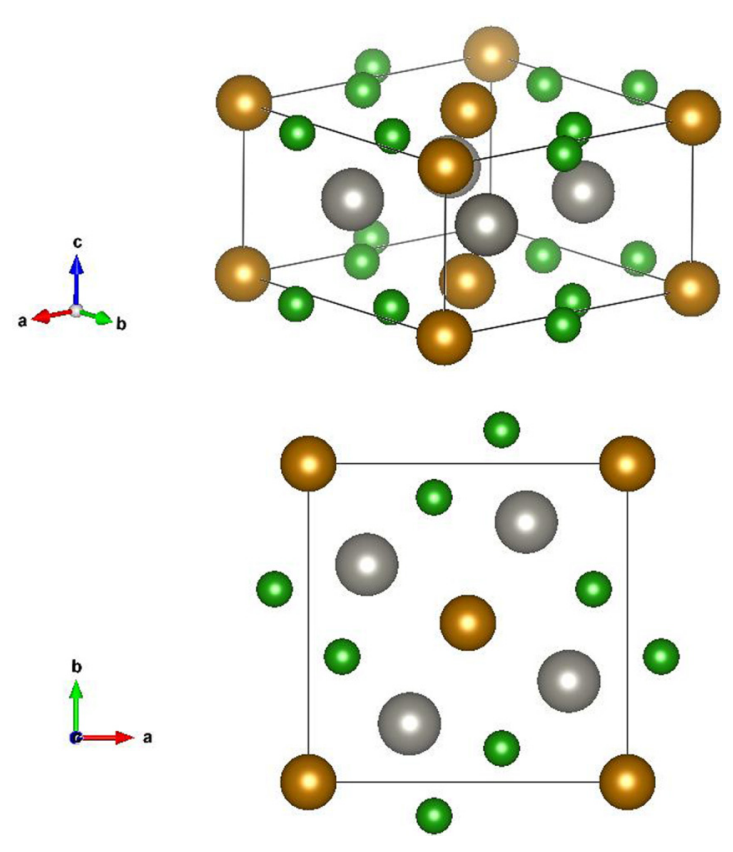

FIG. 4. Tetragonal unit cell of $\mathrm{W}_{2} \mathrm{FeB}_{2}$. Iron atoms are shown with brown spheres, $\mathrm{B}$ atoms are green, and $\mathrm{W}$ atoms are grey.

To address the stability of $\mathrm{Pt}_{2} \mathrm{FeNi}$ as a bulk compound we analyzed theoretically the phase stability. Its formation enthalpy was found to be $\Delta H=-0.19 \mathrm{eV} /$ f.u., which indicates the bulk crystalline phase to be stable. From the three materials in Table II $\mathrm{Pt}_{2} \mathrm{FeNi}$ has the best average performance with a MAE above $2 \mathrm{MJ} / \mathrm{m}^{3}$ and a saturation magnetization of $1 \mathrm{~T}$. Its Curie temperature is still below room temperature, but it might be increased, e.g., by alloying or doping. The formation enthalpy of the final set of materials can be found in Table VI.

Density of states for the three materials listed in Table II, are plotted in Figs. 5 and $6 . \mathrm{Pt}_{2} \mathrm{FeCu}$ and $\mathrm{Pt}_{2} \mathrm{FeNi}$, which have the similar magnetic moments, have the similar DOS, with the spin-up channel almost completely filled and the spin-down channel partially occupied. In these compounds it is possible to discern from the partial DOS, hybridization between $3 d$ orbitals of $\mathrm{Fe}$ and $\mathrm{Ni}(\mathrm{Cu})$ and $5 d$ orbitals of Pt. For $\mathrm{W}_{2} \mathrm{FeB}_{2}$ hybridization between $3 d$ and $5 d$ orbitals is also obvious. However, for this compound the exchange splitting is not as marked as for $\mathrm{Pt}_{2} \mathrm{FeCu}$ and $\mathrm{Pt}_{2} \mathrm{FeNi}$, and both spin-channels are partially filled.

From Table II it becomes clear that two of the three compounds are not suitable for applications that require larger volume or mass since they contain $\mathrm{Pt}$, which is expensive. In fact, from the price-performance viewpoint, of the materials listed in Table II, $\mathrm{W}_{2} \mathrm{FeB}_{2}$ has the highest potential for bulk applications in large volume or mass, although one would have to find the ways to increase the ordering temperature and preferably also the saturation moment.

\section{B. Post high-throughput refinement}

Building on the high throughput study presented in the previous section the outcome can be used to further improve the magnetic properties by careful analysis and modification of the existing phases. Here we focus on the tetragonal $\mathrm{Pt}_{2} \mathrm{MM}^{\prime}$ phase. One may speculate that isoelectronic versions
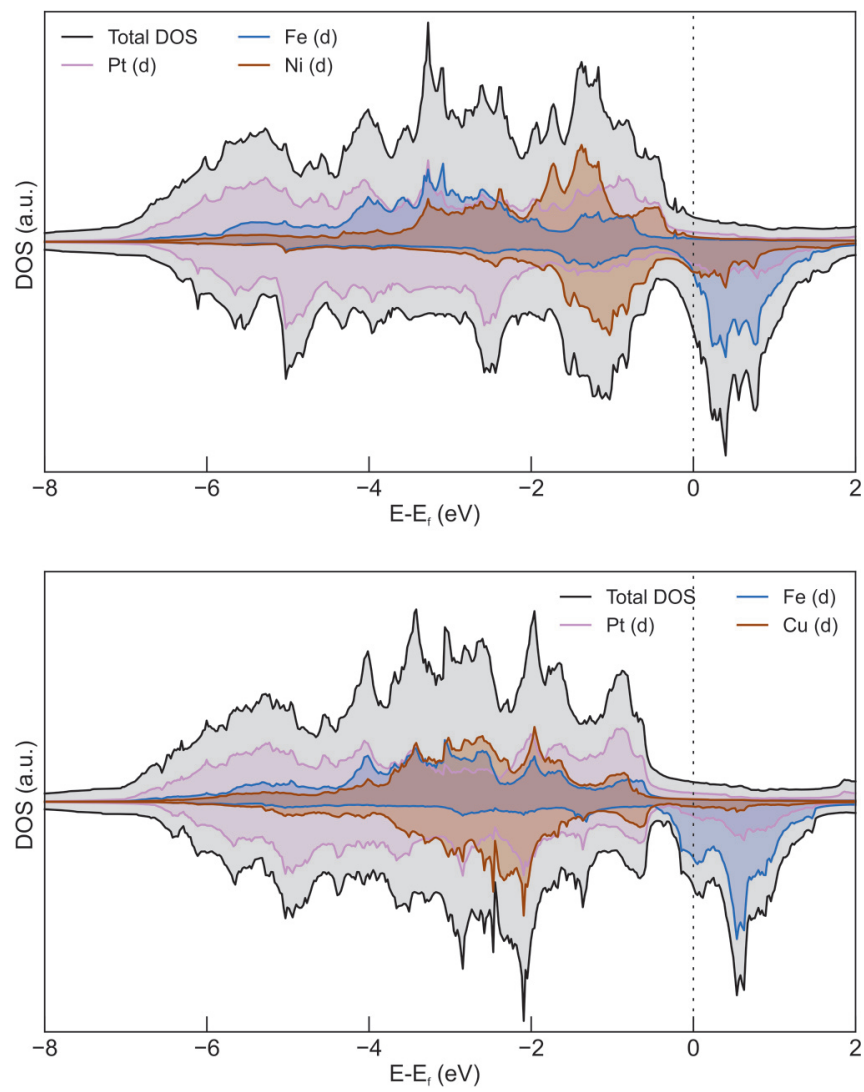

FIG. 5. Calculated spin-polarized density of states of the experimental crystal structures of $\mathrm{Pt}_{2} \mathrm{FeNi}$ (top) and $\mathrm{Pt}_{2} \mathrm{FeCu}$ (bottom). Only the $d$-states are shown with spin-up density of states at the top and spin-down at the bottom.

of them would also be good candidates as permanent magnets. Typically the chemistry of isoelectronic elements is similar, and for this reason a substitution of such elements may result in similar phase and structural stability. It is possible that alloying of $\mathrm{Ni}$ and $\mathrm{Pd}$ for $\mathrm{Pt}$ in $\mathrm{Pt}_{2} \mathrm{FeNi}$ and $\mathrm{Pt}_{2} \mathrm{FeCu}$ may give materials with desired magnetic properties for permanent magnets. In particular, Ni substitution is desired to reduce the cost of the compound.

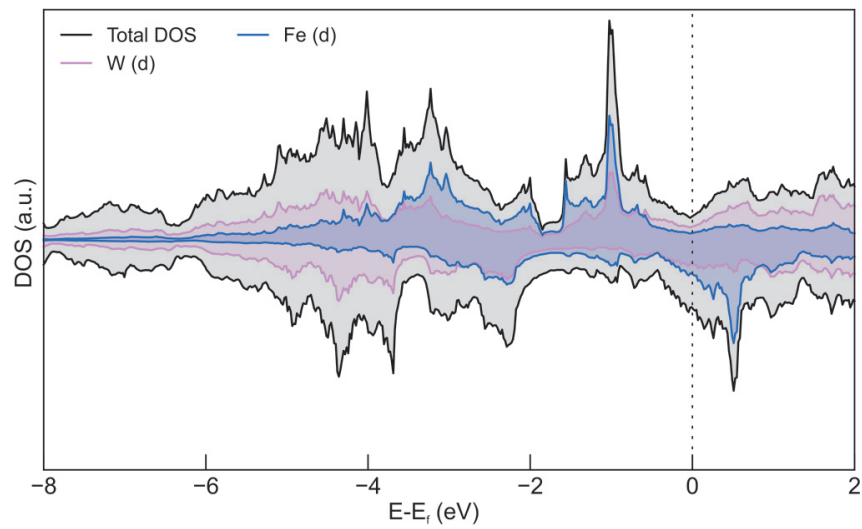

FIG. 6. Calculated spin-polarized density of states of the experimental crystal structure of $\mathrm{W}_{2} \mathrm{FeB}_{2}$, spin-up (top) and spin-down (bottom). Only the $d$-states are shown with spin-up density of states at the top and spin-down at the bottom. 
TABLE III. Calculated MAE, saturation magnetization, Curie temperature, and magnetic hardness parameter of the materials proposed based on the findings of high-throughput run.

\begin{tabular}{lcccccc}
\hline \hline Material & $\begin{array}{c}\text { Space } \\
\text { group }\end{array}$ & $\begin{array}{c}\text { Volume/ion } \\
\AA^{3}\end{array}$ & $\begin{array}{c}\mathrm{MAE} \\
\mathrm{MJ} / \mathrm{m}^{3}\end{array}$ & $\begin{array}{c}\text { Sat. magn. } \\
\mathrm{T}\end{array}$ & $\begin{array}{c}T_{\mathrm{C}} \\
\mathrm{K}\end{array}$ & $\kappa$ \\
\hline $\mathrm{Pt}_{2} \mathrm{CoNi}$ & 123 & 13.47 & 5.46 & 0.83 & 385 & 3.16 \\
$\mathrm{Pt}_{2} \mathrm{FeCo}$ & 123 & 13.75 & 6.83 & 1.24 & 605 & 2.36 \\
\hline \hline
\end{tabular}

At the same time one may try to resolve the issue of the low Curie temperature obtained for $\mathrm{Pt}_{2} \mathrm{FeNi}$ and $\mathrm{Pt}_{2} \mathrm{FeCu}$ (which otherwise have sufficiently high MAE and saturation magnetization) by replacing either of the $3 d$ metals with a different element. For that reason we decided to look into similar compounds that contain cobalt, which usually increases the $T_{\mathbf{C}}$. $\mathrm{Pt}_{2} \mathrm{CoNi}$ and $\mathrm{Pt}_{2} \mathrm{FeCo}$ are not in the current version of ICSD that was used here. However, they have been synthesized in the form of nanoparticles [77], which suggests that bulk versions of these systems might be possible. We relaxed these materials in the same space group as $\mathrm{Pt}_{2} \mathrm{FeNi}$ and $\mathrm{Pt}_{2} \mathrm{FeCu}$ and found them to be ferromagnetic. Saturation magnetization, MAE, and other properties of the Co-based materials can be found in Table III, formation enthalpies are given in Appendix B, Table VI. With the Curie temperature increased by the addition of cobalt above the value that is required for a good permanent magnet; $\mathrm{Pt}_{2} \mathrm{CoNi}$ and $\mathrm{Pt}_{2} \mathrm{FeCo}$ represent the best set of properties out of the materials we found after a high-throughput search and screening.

\section{DISCUSSION AND CONCLUSION}

We propose using a materials-specific screening method combined with a high-throughput approach of the electronic structure calculations for the search of possible candidates for rare-earth free permanent magnets. To test the method we performed a data-mining search among the known crystal structures of the ICSD [28] containing $3 d$ and $5 d$ elements. A number of materials have been found, in particular three promising compounds which have previously not been studied with respect to the magnetic anisotropy. The three systems identified have magnetic properties that satisfy the requirements of having a high saturation magnetization and a large MAE, necessary for a material to be used as a highperformance permanent magnet. The identified compounds are $\mathrm{Pt}_{2} \mathrm{FeNi}, \mathrm{Pt}_{2} \mathrm{FeCu}$, and $\mathrm{W}_{2} \mathrm{FeB}_{2}$. GaMnPt and $\mathrm{Mn}_{2} \mathrm{Ta}$ were found to have a complex magnetic ground state which is not desirable for permanent magnet applications but might have potential for other magnetic applications.

Having only the MAE in mind, the $\mathrm{Pt}_{2} \mathrm{FeCu}$ system is the most favorable. However, as can be seen from Table II the Curie temperature is very low $(30 \mathrm{~K})$ whereas the $T_{\mathbf{C}}$ of the sister compound $\mathrm{Pt}_{2} \mathrm{FeNi}$ is $230 \mathrm{~K}$. Thus replacing $\mathrm{Cu}$ by a magnetic element improves the transition temperature. We investigated the sister compounds $\mathrm{Pt}_{2} \mathrm{CoNi}$ and $\mathrm{Pt}_{2} \mathrm{FeCo}$ which, although not yet in the ICSD database, have been synthesized in the form of nanoparticles [77]. Replacing some of the elements by cobalt indeed increased the $T_{\mathrm{C}}$ to $385 \mathrm{~K}$ for bulk $\mathrm{Pt}_{2} \mathrm{CoNi}$ and $605 \mathrm{~K}$ for $\mathrm{Pt}_{2} \mathrm{FeCo}$, while keeping the high MAE and saturation magnetization. Hence $\mathrm{Pt}_{2} \mathrm{CoNi}$ and
$\mathrm{Pt}_{2} \mathrm{FeCo}$ possess the best set of properties out of the materials we found after a high-throughput search and screening.

We would like to note that even though our calculations show GaMnPt to be noncollinear, it has been reported in the literature to be ferromagnetic with the $T_{\mathbf{C}}$ around $220 \mathrm{~K}$ and saturation magnetization of $3.15 \mu_{B}$ per formula unit (measurements at $4.2 \mathrm{~K}$ ) [72]. Our ferromagnetic RSPT calculations produce a value of $3.8 \mu_{B}$ per f.u., i.e., a slightly larger value. The theory was made for a defect free sample, which might explain part of the small difference between theory and experiment. If the ferromagnetic phase of $\mathrm{GaMnPt}$ can indeed be stabilized, it is also a promising candidate for a rare-earth free permanent magnet demonstrating a combination of high MAE and saturation magnetization. However, the $T_{\mathbf{C}}$ will need to be increased in order for this material to be relevant for room temperature applications.

The clear drawback of the materials found is the high $\mathrm{Pt}$ concentration. Mostly, Pt-based materials will not be able to compete with rare-earth magnets in terms of price. However, these systems may be relevant for applications that do not rely on large samples, e.g., as write heads. Furthermore, as discussed in the previous section isoeletronic partial replacement of Pt might be a solution but is still under consideration and beyond the scope of the present paper. Out of the five listed compounds, the most interesting one for applications using larger masses or volumes, seems to be $\mathrm{W}_{2} \mathrm{FeB}_{2}$ which has a high $\mathrm{MAE}=1.07 \mathrm{MJ} / \mathrm{m}^{3}$, although its saturation magnetization is slightly too low to meet the requirement of a good permanent magnet; it is found to be $0.40 \mathrm{~T}$. Further alloying to increase the saturation moment and ordering temperature would make this system even more competitive from an application point of view.

Furthermore, Mo and $\mathrm{Cr}$ substitution for $\mathrm{W}$ in $\mathrm{W}_{2} \mathrm{FeB}_{2}$ could be an interesting avenue to search for rare-earth free permanent magnets. Since the calculated ordering temperature of this compound is only $165 \mathrm{~K}$ (Table II), the Cr substitution (which is an element that traditionally has a larger interatomic exchange interaction than $\mathrm{W}$ ), may be a way to increase the $T_{\mathbf{C}}$. We hope that at some point the materials we have found would be investigated experimentally to test our theoretical predictions.

This first application of a high-throughput search of a limited part of the Inorganic Crystal Structure Database suggests that it possible to find candidates for rare-earth free permanent magnets using a data-mining approach. Further steps in this search would be to release some of the criteria used here, e.g., the limitation of only using $3 d$ and $5 d$ elements, and a maximum of three elements for a suitable compound. Such investigations are underway. We also demonstrated that a post analysis of the output of the high throughput study can be used to further optimize the magnetic properties and identify novel phases.

\section{ACKNOWLEDGMENTS}

The authors would like to acknowledge the support of the Swedish Foundation for Strategic Research, the Swedish Energy Agency, the Swedish Research Council, The Knut and Alice Wallenberg Foundation, eSSENCE, STandUPP and the CSC IT Centre for Science, and the Swedish National 
Infrastructure for Computing (SNIC) for the computation resources.

\section{APPENDIX A: MATERIALS DISCARDED DURING THE FINAL STEPS}

During the final steps of the high-throughput search we discarded a number of materials that either did not appear to be ferromagnetic or had a planar magnetic anisotropy, according to our calculations. The list of these materials can be found below (Table IV). It doesn't contain the systems with magnetic moment being too small for a strong permanent magnet or material whose magnetic state has been known before.

TABLE IV. Materials that were considered more thoroughly after the high-throughput step, which, however, did not fulfill the requirements of a good permanent magnet. NM stands for nonmagnetic, NC means noncollinear magnetic ordering

\begin{tabular}{|c|c|c|c|c|c|}
\hline Material & $\begin{array}{l}\text { ICSD } \\
\text { number }\end{array}$ & $\begin{array}{l}\text { Space } \\
\text { group }\end{array}$ & $\begin{array}{l}\text { Mag. } \\
\text { state }\end{array}$ & $\begin{array}{c}\mathrm{MAE}(\mathrm{th}) \\
\mathrm{MJ} / \mathrm{m}^{3}\end{array}$ & $\begin{array}{c}\text { Sat. magn. } \\
\mathrm{T}\end{array}$ \\
\hline $\mathrm{Al}_{8} \mathrm{Fe}_{4} \mathrm{Hf}$ & 607535 & 139 & FM & -1.4 & 0.28 \\
\hline $\mathrm{Fe}_{7} \mathrm{~W}_{6}$ & 634060 & 166 & FM & -4.6 & 0.63 \\
\hline CoHfSn & 623801 & 189 & FM & -0.65 & 0.18 \\
\hline $\mathrm{Mn}_{2} \mathrm{Ta}$ & 109357 & 194 & $\mathrm{NC}$ & 1.65 & 0.54 \\
\hline $\mathrm{GaMnPt}$ & 103807 & 194 & $\mathrm{NC}$ & 3.80 & 0.95 \\
\hline $\mathrm{FeHfCl}_{6}$ & 39817 & 163 & AFM & & \\
\hline GeMnTa & 637098 & 189 & AFM & & \\
\hline $\mathrm{FeTa}_{2} \mathrm{~B}_{2}$ & 614207 & 127 & AFM & & \\
\hline $\mathrm{W}_{2} \mathrm{CoB}_{2}$ & 16776 & 71 & AFM & & \\
\hline $\mathrm{Co}_{2} \mathrm{Ta}$ & 108151 & 194 & AFM & & \\
\hline $\mathrm{W}_{2} \mathrm{MnB}_{2}$ & 44449 & 127 & NM & & \\
\hline $\mathrm{W}_{2} \mathrm{NiB}_{2}$ & 615069 & 71 & NM & & \\
\hline $\mathrm{Ta}_{4} \mathrm{FeP}$ & 86378 & 124 & NM & & \\
\hline $\mathrm{FeGa}_{2} \mathrm{Hf}_{6}$ & 631770 & 189 & NM & & \\
\hline $\mathrm{Hf}_{6} \mathrm{CoBi}_{2}$ & 54566 & 189 & NM & & \\
\hline $\mathrm{CoGa}_{2} \mathrm{Hf}_{6}$ & 623077 & 189 & NM & & \\
\hline CoGaHf & 623085 & 189 & NM & & \\
\hline
\end{tabular}

\section{APPENDIX B: ADDITIONAL STRUCTURAL INFORMATION AND FORMATION ENTHALPIES FOR THE MATERIALS FOUND SUITABLE TO BE USED AS PERMANENT MAGNETS}

During the high-throughput search three materials were found to be suitable candidates to be used as rare-earth free permanent magnets: $\mathrm{Pt}_{2} \mathrm{FeNi}, \mathrm{Pt}_{2} \mathrm{FeCu}$, and $\mathrm{W}_{2} \mathrm{FeB}_{2}$; two materials were suggested during the post-refinement step: $\mathrm{Pt}_{2} \mathrm{CoNi}, \mathrm{Pt}_{2} \mathrm{FeCo}$. Below we provide some additional structural information for these materials (Table $\mathrm{V}$ ) as well as their formation enthalpies (Table VI).

TABLE V. Additional structural information (ICSD number, space group, crystal system, cell volume, and magnetic moment of the $3 d$ element) for the three materials that previously have not been investigated for the magnetocrystalline anisotropy (apart from $\mathrm{Pt}_{2} \mathrm{FeNi}$ that which was studied in the form of $\mathrm{L} 1_{0}$ alloy films [71]).

\begin{tabular}{|c|c|c|c|c|c|}
\hline Material & $\begin{array}{c}\text { ICSD } \\
\text { number }\end{array}$ & $\begin{array}{l}\text { Space } \\
\text { group }\end{array}$ & $\begin{array}{l}\text { Crystal } \\
\text { system }\end{array}$ & $\begin{array}{c}\text { Cell } \\
\text { volume, } \\
\AA^{3}\end{array}$ & $\begin{array}{c}\mathrm{M} \\
\text { of } 3 \mathrm{~d}-\mathrm{el} . \\
\mu_{B}\end{array}$ \\
\hline $\mathrm{Pt}_{2} \mathrm{FeNi}$ & 42564 & 123 & tetragonal & 53.83 & $\begin{array}{l}3.21(\mathrm{Fe}) \\
0.86(\mathrm{Ni})\end{array}$ \\
\hline $\mathrm{Pt}_{2} \mathrm{FeCu}$ & 53259 & 123 & tetragonal & 54.16 & $\begin{array}{l}3.17(\mathrm{Fe}) \\
0.12(\mathrm{Cu})\end{array}$ \\
\hline $\mathrm{W}_{2} \mathrm{FeB}_{2}$ & 43016 & 127 & tetragonal & 102.37 & $1.66(\mathrm{Fe})$ \\
\hline
\end{tabular}

TABLE VI. Formation enthalpies for the final systems with respect to the binary components and elemental components, eV/f.u.

\begin{tabular}{|c|c|c|c|}
\hline Material & $\begin{array}{l}\text { Corresp. } \\
\text { binaries }\end{array}$ & $\begin{array}{c}\Delta H \\
\text { (wrt binary comp.) } \\
\text { eV/f.u. }\end{array}$ & $\begin{array}{c}\Delta H \\
\text { (wrt elem. comp.) } \\
\text { eV/f.u. }\end{array}$ \\
\hline $\mathrm{Pt}_{2} \mathrm{FeNi}$ & $\mathrm{FePt}, \mathrm{NiPt}$ & -0.186 & -0.829 \\
\hline $\mathrm{Pt}_{2} \mathrm{FeCu}$ & $\mathrm{FePt}, \mathrm{CuPt}$ & -4.43 & -2.57 \\
\hline $\mathrm{W}_{2} \mathrm{FeB}_{2}$ & $\mathrm{FeB}, \mathrm{Fe}_{2} \mathrm{~W}, \mathrm{Fe}$ & -0.398 & -2.07 \\
\hline $\mathrm{Pt}_{2} \mathrm{CoNi}$ & $\mathrm{CoPt}, \mathrm{NiPt}$ & 0.364 & -0.023 \\
\hline $\mathrm{Pt}_{2} \mathrm{FeCo}$ & $\mathrm{CoPt}, \mathrm{FePt}$ & 0.232 & -0.420 \\
\hline
\end{tabular}

[1] D. J. Craik, Magnetism: Principles and Applications (Wiley, New York, 1995).

[2] J. M. D. Coey, Rare-Earth Iron Permanent Magnets, Springer Series in Solid State Science (Clarendon, Oxford, 1996).

[3] R. Skomski and J. M. D. Coey, Permanent Magnetism (Institute of Physics, Bristol, England, 1999).

[4] B. Balamurugan, B. Das, W. Y. Zhang, R. Skomski, and D. J. Sellmyer, J. Phys.: Condens. Matter 26, 064204 (2014).

[5] V. Ly, X. Wu, L. Smillie, T. Shoji, A. Kato, A. Manabe, and K. Suzuki, J. Alloys Compd. 615, S285 (2014).

[6] M. D. Kuz'min, K. P. Skokov, H. Jian, I. Radulov, and O. Gutfleisch, J. Phys.: Condens. Matter 26, 064205 (2014).
[7] B. Balasubramanian, B. Das, R. Skomski, W. Y. Zhang, and D. J. Sellmyer, Adv. Mater. 25, 6090 (2013).

[8] E. Lottini, A. López-Ortega, G. Bertoni, S. Turner, M. Meledina, G. Van Tendeloo, C. de Julián Fernández, and C. Sangregorio, Chem. Mater. 28, 4214 (2016).

[9] B. Balamurugan, B. Das, V. R. Shah, R. Skomski, X. Z. Li, and D. J. Sellmyer, Appl. Phys. Lett. 101, 122407 (2012).

[10] E. Anagnostopoulou, B. Grindi, L.-M. Lacroix, F. Ott, I. Panagiotopoulos, and G. Viau, Nanoscale 8, 4020 (2016).

[11] T. R. Gao, Y. Q. Wu, S. Fackler, I. Kierzewski, Y. Zhang, A. Mehta, M. J. Kramer, and I. Takeuchi, Appl. Phys. Lett. 102, 022419 (2013). 
[12] T. J. Nummy, S. P. Bennett, T. Cardinal, and D. Heiman, Appl. Phys. Lett. 99, 252506 (2011).

[13] A. G. Kusne, T. Gao, A. Mehta, L. Ke, M. C. Nguyen, K.-M. Ho, V. Antropov, C.-Z. Wang, M. J. Kramer, C. Long, and I. Takeuchi, Sci. Rep. 4, 6367 (2014).

[14] T. Burkert, L. Nordström, O. Eriksson, and O. Heinonen, Phys. Rev. Lett. 93, 027203 (2004).

[15] G. Andersson, T. Burkert, P. Warnicke, M. Björck, B. Sanyal, C. Chacon, C. Zlotea, L. Nordström, P. Nordblad, and O. Eriksson, Phys. Rev. Lett. 96, 037205 (2006).

[16] J. H. Park, Y. K. Hong, S. Bae, J. J. Lee, J. Jalli, G. S. Abo, N. Neveu, S. G. Kim, C. J. Choi, and J. G. Lee, J. Appl. Phys. 107, 09A731 (2010).

[17] H. Fang, S. Kontos, J. Ångström, J. Cedervall, P. Svedlindh, K. Gunnarsson, and M. Sahlberg, J. Solid State Chem. 237, 300 (2016)

[18] T. Kojima, M. Ogiwara, M. Mizuguchi, M. Kotsugi, T. Koganezawa, T. Ohtsuki, T.-Y. Tashiro, and K. Takanashi, J. Phys.: Condens. Matter 26, 064207 (2014).

[19] X. Zhao, C.-Z. Wang, Y. Yao, and K.-M. Ho, Phys. Rev. B 94, 224424 (2016).

[20] N. V. R. Rao, A. M. Gabay, and G. C. Hadjipanayis, J. Phys. D 46, 062001 (2013).

[21] O. Eriksson, A. Bergman, L. Bergqvist, and J. Hellsvik, Atomistic Spin Dynamics: Foundations and Applications (Oxford University Press, Oxford, 2017).

[22] H. Brooks, Phys. Rev. 58, 909 (1940).

[23] G. C. Fletcher, Proc. Phys. Soc. Sect. A 67, 505 (1954).

[24] J. C. Slonczewski, J. Phys. Soc. Jpn., Suppl. BI 17, 34 (1962).

[25] E. I. Kondorski and E. Straub, Zh. Eksp. Teor. Fiz. 63, 356 (1972).

[26] P. Bruno, Phys. Rev. B 39, 865 (1989).

[27] G. H. O. Daalderop, P. J. Kelly, and M. F. H. Schuurmans, Phys. Rev. B 41, 11919 (1990).

[28] Inorganic crystal structure database, http://www2.fiz-karlsruhe. de/icsd_home.html.

[29] C. C. Fischer, K. J. Tibbetts, D. Morgan, and G. Ceder, Nat. Mater. 5, 641 (2006).

[30] S. Curtarolo, A. N. Kolmogorov, and F. H. Cocks, Calphad 29, 155 (2005).

[31] Y. Saad, D. Gao, T. Ngo, S. Bobbitt, J. R. Chelikowsky, and W. Andreoni, Phys. Rev. B 85, 104104 (2012).

[32] T. D. Sparks, M. W. Gaultois, A. Oliynyk, J. Brgoch, and B. Meredig, Scr. Mater. 111, 10 (2016).

[33] G. Cheon, K.-A. N. Duerloo, A. D. Sendek, C. Porter, Y. Chen, and E. J. Reed, Nano Lett. 17, 1915 (2017).

[34] S. Lebégue, T. Björkman, M. Klintenberg, R. M. Nieminen, and O. Eriksson, Phys. Rev. X 3, 031002 (2013).

[35] T. Tada, S. Takemoto, S. Matsuishi, and H. Hosono, Inorg. Chem. 53, 10347 (2014).

[36] S. Körbel, M. A. L. Marques, and S. Botti, J. Mater. Chem. C 4, 3157 (2016).

[37] M. Klintenberg and O. Eriksson, Comput. Mater. Sci. 67, 282 (2013).

[38] A. N. Kolmogorov, S. Shah, E. R. Margine, A. F. Bialon, T. Hammerschmidt, and R. Drautz, Phys. Rev. Lett. 105, 217003 (2010).

[39] C. Ortiz, O. Eriksson, and M. Klintenberg, Comput. Mater. Sci. 44, 1042 (2009).
[40] M. Klintenberg, J. T. Haraldsen, and A. V. Balatsky, Appl. Phys. Res. 6 (2014).

[41] Q. Gao, I. Opahle, and H. Zhang, Phys. Rev. Mater. 3, 024410 (2019).

[42] S. Sanvito, C. Oses, J. Xue, A. Tiwari, M. Zic, T. Archer, P. Tozman, M. Venkatesan, M. Coey, and S. Curtarolo, Sci. Adv. 3, e1602241 (2017).

[43] S. Curtarolo, G. L. W. Hart, M. Buongiorno Nardelli, N. Mingo, S. Sanvito, and O. Levy, Nat. Mater. 12, 191 (2013).

[44] O. Eriksson, Nat. Nanotech. 13, 180 (2018).

[45] J. M. Wills and B. R. Cooper, Phys. Rev. B 36, 3809 (1987).

[46] J. M. Wills, M. Alouani, P. Andersson, A. Delin, O. Eriksson, and O. Grechnyev, Full-Potential Electronic Structure Method, Springer Series in Solid State Science (Springer, Berlin, 2010).

[47] R. Armiento and A. E. Mattsson, Phys. Rev. B 72, 085108 (2005).

[48] G. Kresse and J. Hafner, Phys. Rev. B 47, 558 (1993).

[49] G. Kresse and J. Hafner, Phys. Rev. B 49, 14251 (1994).

[50] G. Kresse and J. Furthmüller, Comput. Mater. Sci. 6, 15 (1996).

[51] G. Kresse and J. Furthmüller, Phys. Rev. B 54, 11169 (1996).

[52] P. E. Blöchl, Phys. Rev. B 50, 17953 (1994).

[53] J. P. Perdew, K. Burke, and M. Ernzerhof, Phys. Rev. Lett. 77, 3865 (1996).

[54] P. E. Blöchl, O. Jepsen, and O. K. Andersen, Phys. Rev. B 49, 16223 (1994).

[55] H. J. Monkhorst and J. D. Pack, Phys. Rev. B 13, 5188 (1976).

[56] R. Skomski and J. Coey, Scr. Mater. 112, 3 (2016).

[57] A. Ganose, A. Jackson, and D. Scanlon, J. Open Source Software 3, 717 (2018).

[58] J. J. Croat, J. F. Herbst, R. W. Lee, and F. E. Pinkerton, J. Appl. Phys. 55, 2078 (1984).

[59] M. Sagawa, S. Fujimura, N. Togawa, H. Yamamoto, and Y. Matsuura, J. Appl. Phys. 55, 2083 (1984).

[60] K. Strnat, in Handbook of Ferromagnetic Materials, Vol. 4, edited by E. Wohlfarth and K. Buschow (North-Holland, Amsterdam, 1988), pp. 131-209.

[61] J. Trygg, B. Johansson, O. Eriksson, and J. M. Wills, Phys. Rev. Lett. 75, 2871 (1995).

[62] K. Adachi, T. Ido, and K. Sato, Trans. Jpn. Inst. Met. 18, 299 (1977).

[63] P. Eurin and J. Pauleve, IEEE Trans. Magn. 5, 216 (1969).

[64] I. Galanakis, M. Alouani, and H. Dreyssé, Phys. Rev. B 62, 6475 (2000).

[65] W. Grange, I. Galanakis, M. Alouani, M. Maret, J.-P. Kappler, and A. Rogalev, Phys. Rev. B 62, 1157 (2000).

[66] I. V. Solovyev, P. H. Dederichs, and I. Mertig, Phys. Rev. B 52, 13419 (1995).

[67] A. Sakuma, J. Phys. Soc. Jpn. 63, 3053 (1994).

[68] J.-U. Thiele, L. Folks, M. F. Toney, and D. K. Weller, J. Appl. Phys. 84, 5686 (1998).

[69] A. Edström, Phys. Rev. B 96, 064422 (2017).

[70] P. Villars and L. Calvet, Pearson's Handbook of Crystallographic Data for Intermetallic Phases (American Society for Metals, Metals Park, OH, 1985).

[71] R. Montsouka, J. Arabski, A. Derory, J. Faerber, G. Schmerber, and V. Pierron-Bohnes, Mater. Sci. Eng. B 126, 236 (2006). 
[72] K. H. J. Buschow and P. G. van Engen, Phys. Status Solidi A 76, 615 (1983).

[73] K. Buschow and D. D. Mooij, J. Less-Common Met. 99, 125 (1984).

[74] F. A. Hames and J. Crangle, J. Appl. Phys. 42, 1336 (1971).
[75] T. Kanomata, K. Shirakawa, and T. Kaneko, Phys. Status Solidi A 97, K149 (1986).

[76] C. Goyhenex, R. Montsouka, M. Kozłowski, and V. PierronBohnes, Solid State Phenom. 129, 59 (2007).

[77] M. T. Nguyen, R. H. Wakabayashi, M. Yang, H. D. Abruña, and F. J. DiSalvo, J. Power Sources 280, 459 (2015). 